\title{
Optimal filling factor of nanorod lenses for subwavelength imaging
}

\author{
Sergey Yu. Kosulnikov, ${ }^{1}$ Elizaveta A. Yankovskaya, ${ }^{1}$ Stanislav I. Maslovski, ${ }^{2}$ Pavel A. Belov, ${ }^{1,3}$ and Yuri S. Kivshar ${ }^{1,4}$ \\ ${ }^{1}$ Department of Photonics and Optoinformatics, St. Petersburg National Research University of Information Technologies, \\ Mechanics and Optics, Kronverkskiy pr., 49, 197101 St. Petersburg, Russia \\ ${ }^{2}$ Department of Electrical Engineering, Instituto de Telecomunicações, Universidade de Coimbra, Pólo II, 3030-290 Coimbra, Portugal \\ ${ }^{3}$ Queen Mary College, University of London, Mile End Road, London E1 4NS, United Kingdom \\ ${ }^{4}$ School of Electronic Engineering and Computer Science, Nonlinear Physics Centre, Australian National University, Canberra, \\ Australian Capital Territory 0200, Australia
}

(Received 23 August 2011; published 12 December 2011)

\begin{abstract}
We analyze the effect of the filling factor on the imaging performance of metallic nanorod lenses. We observe that thicker nanorods allow lower reflection in the canalization regime and we find optimal values of the filling factor to achieve a transfer function with the characteristics of a perfect lens in a wide range of spatial frequencies.
\end{abstract}

DOI: 10.1103/PhysRevA.84.065801

PACS number(s): 42.79.-e, 78.67.Qa, 07.60.-j

Wire media were suggested and have been extensively studied as the basis for subwavelength imaging devices [1-10]. Arrays of parallel wires can transport near-field images with subwavelength resolution to distances up to several wavelengths, possibly avoiding obstacles [4]. Tapered wire arrays [5-7] can be employed for magnification of such images. Employing arrays of metallic wires of different radii, it is possible to achieve subwavelength imaging in a wide spectrum of electromagnetic waves ranging from radiowaves and microwaves [9] to terahertz frequencies $[6,8]$ or to extend this range to optics using plasmonic nanorods.

The main operational principle of the wire lenses is based on the conversion of the whole spatial spectrum of a near-field source (including the evanescent modes) into the propagating eigenmodes of an array of metallic rods. This principle was confirmed numerically and experimentally $[6-8,11]$. The influence of the unit-cell geometry on the operation of nanowire lenses was recently studied in Ref. [12]. However, the effect of the metal filling factor on the efficiency of the subwavelength imaging has not yet received enough attention. The filling factor in a nanowire lattice may be defined as $f_{r}=\pi(r / a)^{2}$, where $r$ is the radius of the nanorods and $a$ is the lattice period. In the lower limit when $r \ll a$ and $f_{r} \rightarrow 0$, the typical thin wire models apply. The main objective of this paper, however, is to study the effects that occur when the relative radius of the nanowires is not small and approaches the upper limit of $r / a=0.5$.

We study numerically a square lattice of nanorods with the following parameters: period $a=2 \mu \mathrm{m}$; the nanorod length $d=15 a$, and the nanowire radius $r$ varying from $0.05 a$ to $0.45 a$. The operating frequency is chosen close to $5 \mathrm{THz}$. Figure 1(a) depicts the unit cell of the nanowire array as modeled in CST MICROWAVE STUDIO [13]. The material of the nanowires is silver, for which the skin depth is $\delta \approx 30 \mathrm{~nm}$ at $5 \mathrm{THz}$. In the supporting theoretical calculations that we also give below, the Ag nanorods with $r>\delta$ are approximated by the perfect electric conductors (PECs) of the same radius and length as the real nanorods [11].

We are interested in the TM polarized electromagnetic waves for which we calculate both the transmission and the reflection coefficients. The magnetic field of the incident electromagnetic wave is polarized along the $z$ axis. The wave

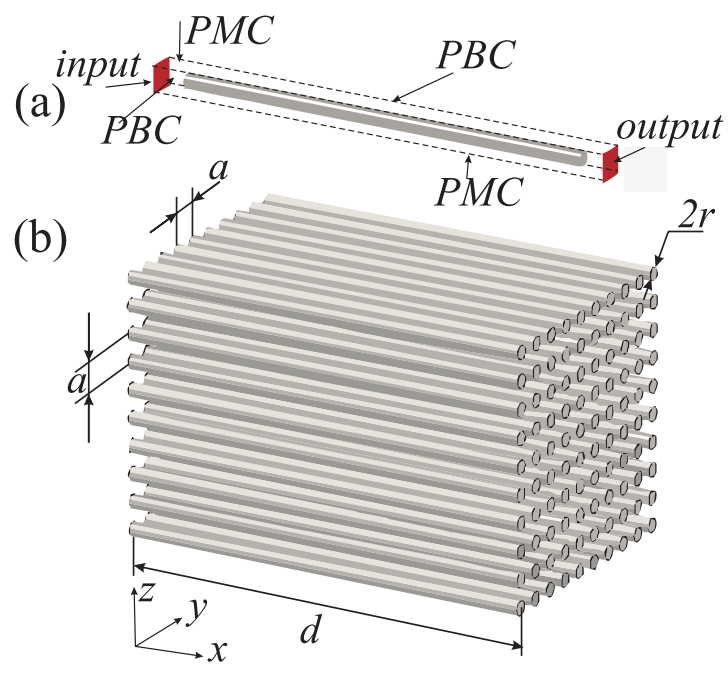

FIG. 1. (Color online) Wire medium: (a) a unit cell and (b) the two-dimensional square lattice of metallic nanorods as modeled by the CST MICROWAVE STUDIO. Notation: PMC, perfect magnetic conductor boundaries; PBC, periodic boundary conditions.

propagates in the $x y$ plane. Inside the nanorod slab, a TM polarized incident wave excites both TM and $\mathrm{TEM}_{x}$ waves (the latter are the waves for which $E_{x}=H_{x}=0$ ). The reflection and transmission properties of the layer of nanorods are presented in Figs. 2-6.

As one can see from Figs. 2(a) and 2(b), if the lens is tuned for the first Fabry-Pérot resonance at $k d / \pi=1$, then the radius variation does not affect the transfer function much. The variation in the transmission coefficient in this case is less than $20 \%$ in the whole range of radii. However, the reflection coefficient $R$ varies significantly with the filling factor. For instance, when $r / a=0.05$, the maximum value of the reflection coefficient seen in Fig. 2(a) is about 0.3 [except at the point $k_{y} d / \pi=1$, which corresponds to the grazing incidence at which, theoretically, $R \rightarrow 1$ and $T \rightarrow 0$; this is clearly seen in Figs. 2(a) and 2(b), where we use a larger number of $k_{y} d$ samples than in the rest of the figures]. As the ratio $r / a$ increases up to about 0.20 the reflection coefficient drops down to $R \approx 0.1$. This is undoubtedly a positive development because it shows that by tuning the filling 
factor one can eliminate the reflection from the input interface of the lens almost completely. The source will consequently be much less influenced by the lens in this case. A further increase in the filling factor $\left(r / a=0.3, f_{r}=0.28\right)$ results in a stronger reflection. From the figure it can be concluded that the strongest reflection corresponds to the largest simulated ratio of $r / a=0.45, f_{r}=0.64$. Thus the relative radii in the range $0.2<r / a<0.25$, which correspond to the filling factor in the range $0.12<f_{r}<0.20$, are optimal in the sense of minimizing reflection. This is a nontrivial fact given that in many works it has been assumed that the reflection grows monotonically with the metal filling factor.

When the lens is tuned slightly below the Fabry-Pérot resonance, the transfer function may stay close to unity or increase monotonically in a wide range of spatial frequencies. This behavior is seen in Figs. 3(a) and 3(b), which are plotted at $k d / \pi=0.98$. The situation illustrated by Fig. 3(a) corresponds to the so-called canalization regime of operation of a wire lens, while the growth of the transfer function seen in Fig. 3(b) resembles amplification of the evanescent waves similar to what happens in a slab of Veselago media (a perfect lens). As seen from the plots, by changing the wire radius and the filling ratio of a wire lens one may switch between these modes of operation.

Figures 4(a) and 4(b) show the characteristics of the same lens at a slightly detuned frequency for which $k d / \pi=0.97$. One may observe sharp resonances in $R$ and $T$ in the case of small relative radii that are related to the excitation
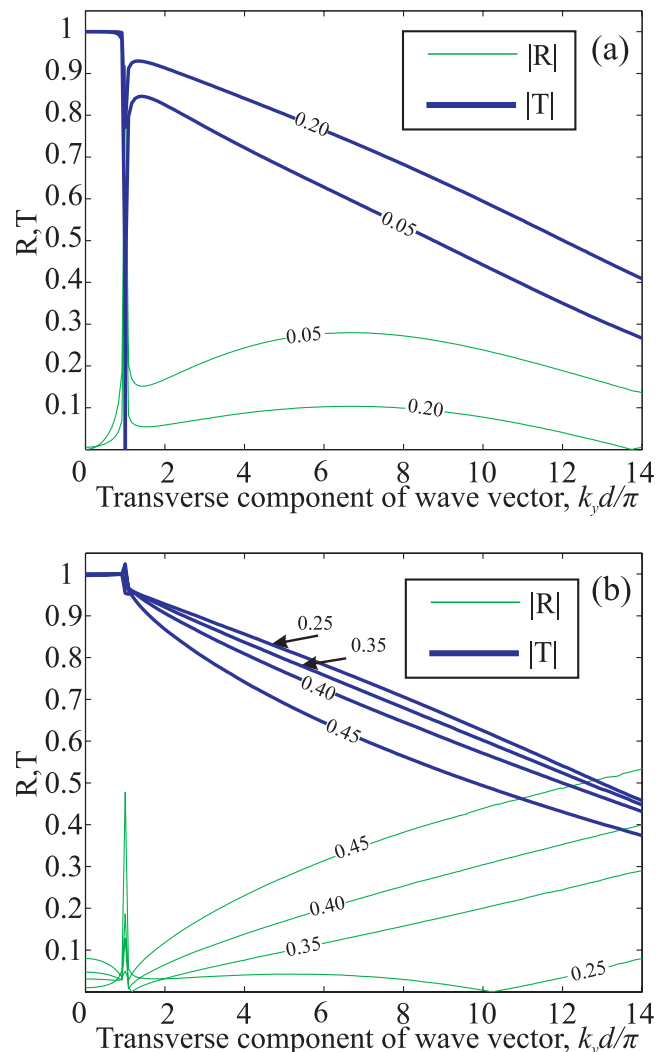

FIG. 2. (Color online) Transmission and reflection coefficients for various values of the relative nanorod radius as functions of the normalized transverse wave number $k_{y} d / \pi$ for $k d / \pi=1.0$ on a linear scale.
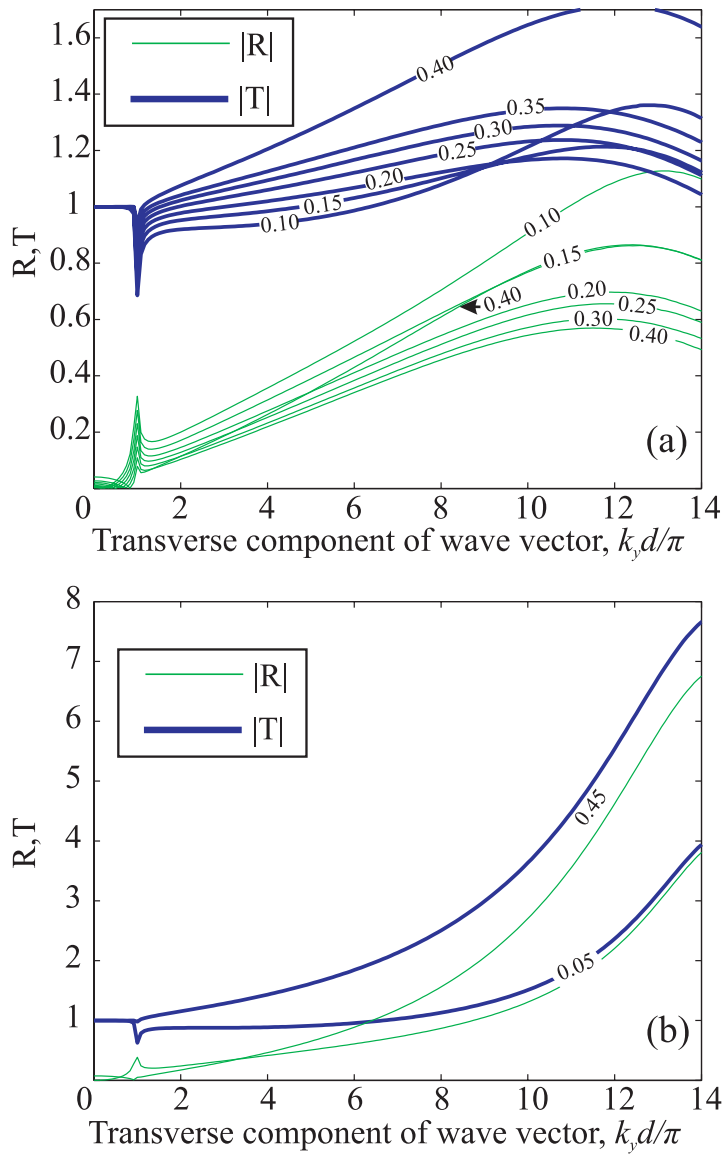

FIG. 3. (Color online) Same as in Fig. 2 for $k d / \pi=0.98$ on a linear scale.

of nonpropagating modes in the layer of nanorods. This effect results in image distortions, as indicated in Ref. [2]. However, if the filling factor is increased the plasmon-polariton resonances may be shifted to higher spatial frequencies or even dumped, as seen from Fig. 4(a). This happens in the range of the relative nanorod radii of $0.25<r / a<0.35$ $\left(0.2<f_{r}<0.38\right)$. Thus the nanowire lenses with thicker nanowires may have a larger available band of operation in the spatial spectrum than the same structures with thin nanowires. A further increase of the filling factor, which is illustrated in Fig. 4(b), leads to the resonances shifted to lower spatial frequencies, which is undesirable.

In Figs. 5(a) and 5(b) the same transmission and reflection coefficients are plotted for the structure with $k d / \pi=0.96$. As in the previous case, one can observe resonances in $R$ and $T$, however, there is no longer a specific range of the radii where the resonances are dumped. Moreover, for the relative nanowire radii values around $0.25\left(f_{r}=0.20\right)$, one may expect a mode of operation resembling (for a limited range of spatial frequencies) a perfect lens, similar to what was discussed above (Fig. 3).

Another interesting peculiarity of the transmission and reflection coefficients [which is also partially seen in Figs. 4(a) and $4(\mathrm{~b})]$ is in their nonmonotonic behavior with the increase of the filling factor. The resonant peaks move to the higher spatial frequencies when the relative radius is changed from 0.05 to $0.20\left(7.8 \times 10^{-3}<f_{r}<0.12\right)$, but a further increase in $r / a$ 

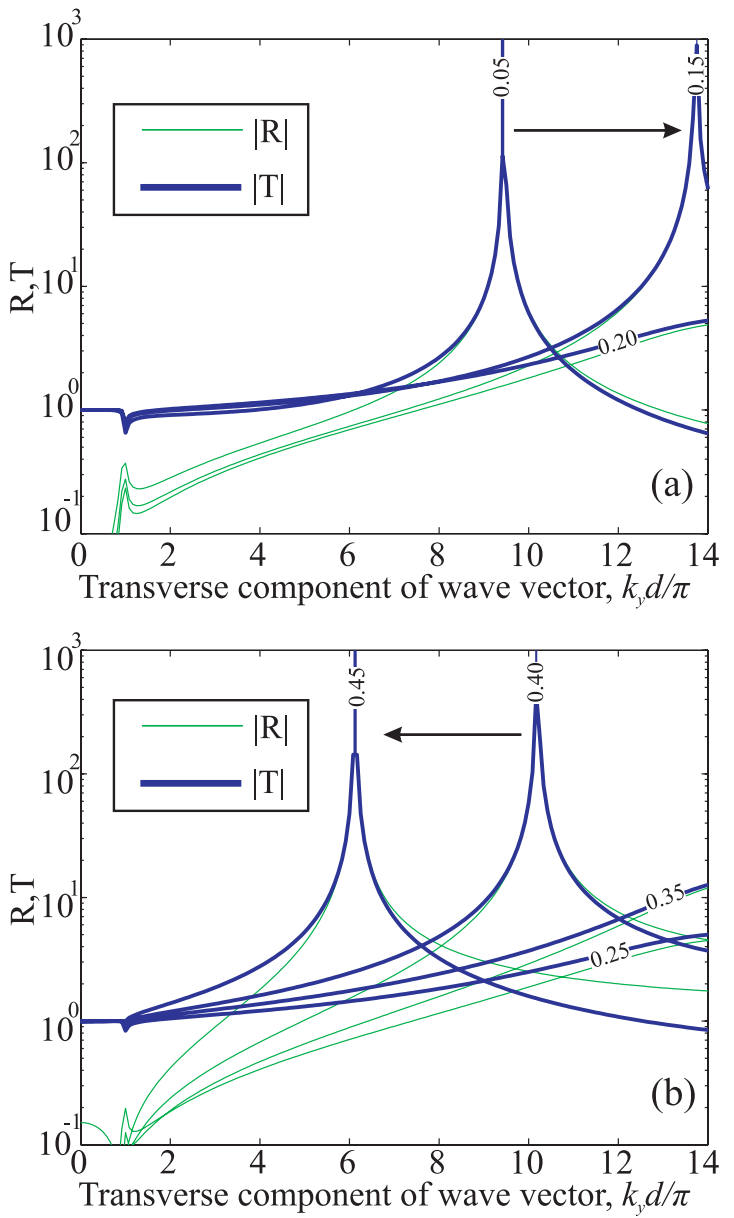

FIG. 4. (Color online) Same as in Fig. 2 for $k d / \pi=0.97$ on a logarithmic scale.

results in the resonances moving to the lower frequencies, as shown in Fig. 6, for $k_{y} d / \pi=0.96$ and 0.97 .

To study this peculiarity we have calculated the reflection and transmission coefficients using the analytical approach developed earlier in Ref. [2]. In this theory the nanowires are modeled as plasmonic cylinders with the boundary conditions being equivalent to a vanishing macroscopically averaged conduction current at the wire tips. In the following calculations the nanorod radius is greater than the skin depth; therefore, we may approximate $\mathrm{Ag}$ nanorods as PECs. We assume the vanishing conduction current condition at the ends of the nanorods. Under these assumptions, the transmission and reflection coefficients coincide with Eqs. (8) and (9) of Ref. [2] and we should use the plasma wave number from Ref. [14] as being applicable to the nanowires of large radii, $\left(k_{p} a\right)^{2}=2 \pi\left\{\log \left[a^{2} / 4 r_{0}\left(a-r_{0}\right)\right]\right\}^{-1}$. However, the theoretical results obtained in this way do not agree with our numerical simulations: The theory predicts a monotonic shift of the resonances with $r$.

This is because the condition at the ends of thick rods was assumed to be the same as in thin wires, for which it was originally derived. However, as was recently found in Ref. [15], the conditions for the macroscopically averaged current at the ends of a conducting rod are $\left[J(x) \pm\left(C_{0} / C\right) d J(x) / d x\right]=0$, where $C$ is the effective capacitance of the rod per unit length
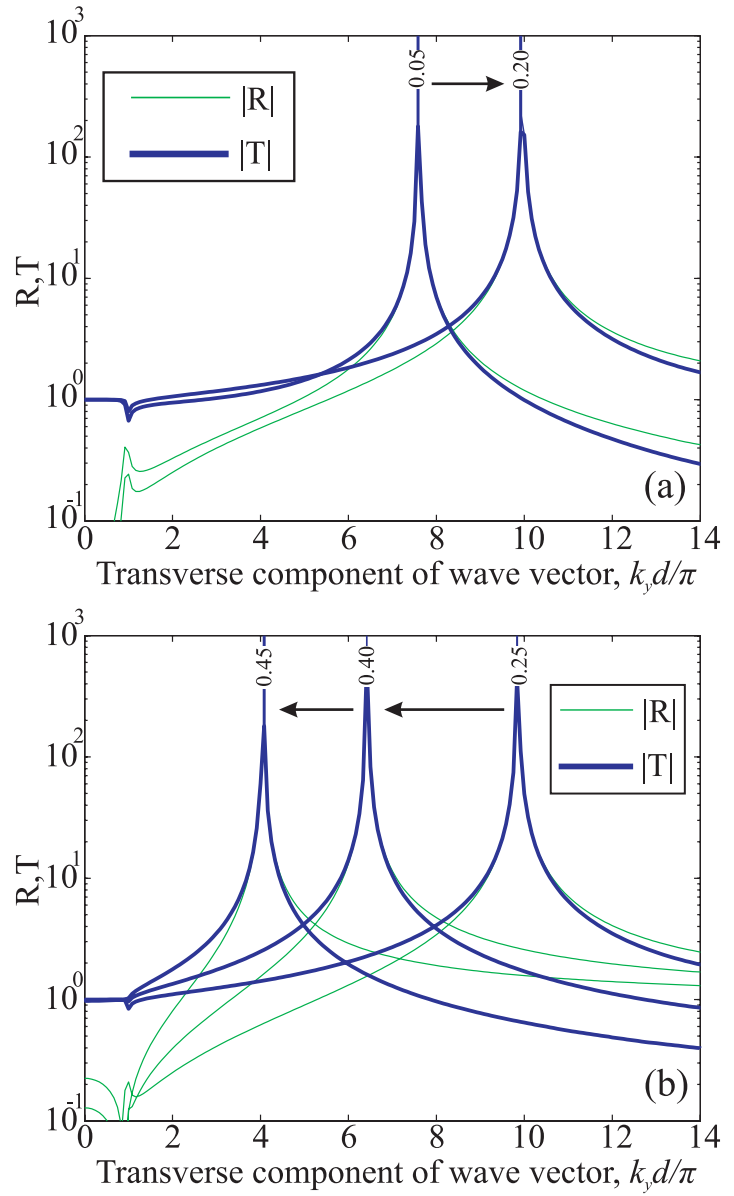

FIG. 5. (Color online) Same as in Fig. 2 for $k d / \pi=0.96$ on a logarithmic scale.

and $C_{0}$ is an additional capacitance associated with the tip of the rod. For thin nanorods, $C_{0} / C \rightarrow 0$ and the usual condition of the vanishing current applies. However, in thick nanorods the charges may be accumulated at the tips due to a finite $C_{0}>0$ and the macroscopically averaged current may not vanish in the same manner as with the thin nanorods.

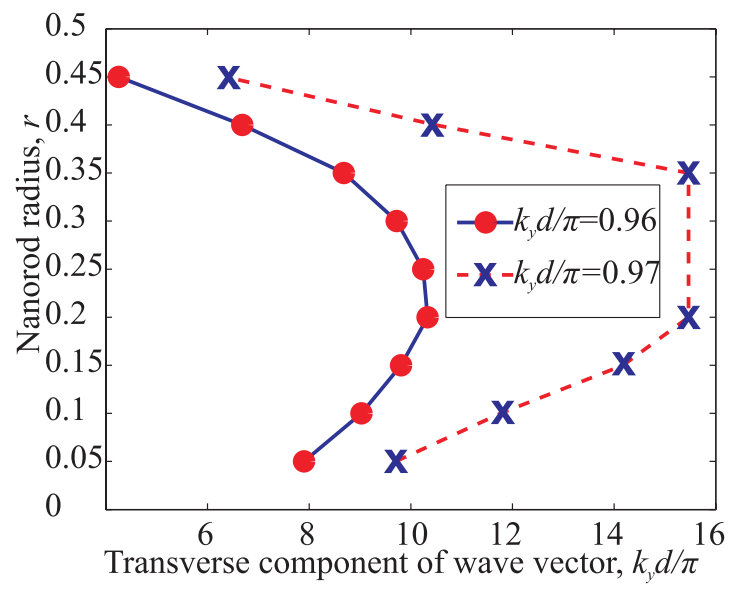

FIG. 6. (Color online) Position of the resonant peak as follows from Figs. 4 and 5. The two curves are for $k_{y} d / \pi=0.96$ and 0.97 . 
With the mentioned condition at the ends of thick nanorods, the nonmonotonic behavior of the resonances observed in the numerical simulations can be qualitatively explained as follows. While the relative nanorod radius remains small enough, the dependence is close to that predicted for the case of zero tip capacitance. In this case one observes the resonances shifting toward the larger values of the spatial frequencies (see Fig. 5), which is related to the monotonic increase of the plasma frequency with the wire radius. However, for the nanowires with $r>0.25 a$, the increase in the tip capacitance becomes dominant, which results in the opposite shift of the effective plasma frequency of the nanorod slab so that its resonances move toward lower spatial frequencies.

In conclusion, we have studied numerically the effect of the filling ratio on the characteristics of the nanorod lenses. We have revealed that there exists a range of relative nanorod radii for which the reflection from the front surface of the lens can be made as small as 0.1 . This corresponds to filling factors in the range $0.12<f_{r}<0.20$. We have studied the properties of the transfer function of the wire lens with thick nanowires and found that a higher filling factor can allow for a broader range of operational spatial frequencies. Our numerical simulations have shown a nonmonotonic dependence of the plasmon resonances on the relative nanowire radius. This effect is attributed to a nonvanishing tip capacitance of the thick nanorods.

The authors acknowledge support from the Ministry of Education and Science of the Russian Federation (Russia), Dynasty Foundation (Russia), EPSRC (United Kingdom), and Australian Research Council (Australia).
[1] P. A. Belov, Y. Hao, and S. Sudhakaran, Phys. Rev. B 73, 033108 (2006).

[2] P. A. Belov and M. G. Silveirinha, Phys. Rev. E 73, 056607 (2006).

[3] P. A. Belov, Y. Zhao, S. Sudhakaran, A. Alomainy, and Y. Hao, Appl. Phys. Lett. 89, 262109 (2006).

[4] A. Rahman, P. A. Belov, Y. Hao, and C. Parini, Opt. Lett. 35, 142 (2010).

[5] P. Ikonen, C. Simovski, S. Tretyakov, P. Belov, and Y. Hao, Appl. Phys. Lett. 91, 104102 (2007).

[6] G. Shvets, S. Trendafilov, J. B. Pendry, and A. Sarychev, Phys. Rev. Lett. 99, 053903 (2007).

[7] S. Kawata, A. Ono, and P. Verma, Nature Photon. 2, 438 (2008).

[8] M. G. Silveirinha, P. A. Belov, and C. R. Simovski, Phys. Rev. B 75, 035108 (2007).
[9] P. A. Belov, Y. Zhao, S. Tse, P. Ikonen, M. G. Silveirinha, C. R. Simovski, S. Tretyakov, Y. Hao, and C. Parini, Phys. Rev. B 77, 193108 (2008).

[10] B. D. F. Casse, W. T. Lu, Y. J. Huang, E. Gultepe, L. Menon, and S. Sridharan, Appl. Phys. Lett. 96, 023114 (2010).

[11] M. G. Silveirinha, P. A. Belov, and C. R. Simovski, Opt. Lett. 33, 1726 (2008).

[12] L. Yi, X. Zhou, Y. Fu, and L. Yang, Plasmonics 6, 281 (2011).

[13] CST MICROWAVE STUDIO $^{\circledR}$ 9.0, CST of America, Inc. [www.cst.com].

[14] S. I. Maslovski and M. G. Silveirinha, Phys. Rev. B 80, 245101 (2009).

[15] S. I. Maslovski, T. A. Morgado, M. G. Silveirinha, C. S. R. Kaipa, and A. B. Yakovlev, New J. Phys. 12, 113047 (2010). 